\title{
Herramienta de segmentación automática de masas forestales a partir de sensores remotos: LiDAR y Sentinel 2
}

\section{Automatic stand delineation tool based on remote sensing: LiDAR and Sentinel 2}

Bezares, F. ${ }^{*} ;$ Bravo, F. ${ }^{1,2}$; Tomé Morán, J.L. ${ }^{3}$

\author{
${ }^{1}$ Universidad de Valladolid, Campus la Yutera. Avda. de Madrid 50, 34004, Palencia, España \\ ${ }^{2}$ Instituto universitario de Investigación en Gestión Forestal Sostenible. \\ Universidad de Valladolid-INIA, Avda. de Madrid 50, 34004, Palencia, España \\ ${ }^{3}$ Agresta S. Coop. Calle del duque Fernán Núñez, 2, 28012 Madrid, España
}




\title{
Resumen
}

La delineación de rodales tradicionalmente se ha basado en cartografía y criterio experto de profesionales forestales. La aparición de sensores remotos permite disponer de información que puede ser utilizada para rodalizar de forma semiautomática. Este estudio busca desarrollar una herramienta para automatizar los procesos de segmentación requeridos en la gestión forestal. Para ello, la herramienta se desarrolla en Python 3 y emplea como fuentes de datos, información de LIDAR aéreo e imágenes de Sentinel 2. La metodología empleada, comienza con el procesado de información LIDAR usando el software de FUSION para obtener información de la estructura de la vegetación. A continuación, se combina un índice de vegetación normalizado, generado a partir de la información satelital, con las imágenes de las variables LiDAR. Como resultado, se obtiene un raster multibanda normalizado. Finalmente, el algoritmo de segmentación de Orfeo Tools recibe el raster multibanda, junto con una serie de parámetros de entrada. El resultado del software es un mapa regiones homogéneas del bosque, que no debe ser considerado como una solución definitiva, sino como un apoyo que el gestor puede emplear para determinar unidades de gestión. Para conseguir mejores resultados de segmentación, cada proyecto y tipo de masa requiere una combinación diferente de parámetros, que ha de ser determinada, para cada tipo de masa. Además del archivo de segmentación, las variables raster y otros sus productos se presentan como resultados. Como conclusión, la herramienta que se presenta es una solución moderna, gratuita, rápida, fiable y basada en datos abiertos que combina algoritmos para automatizar las etapas iniciales de un plan de gestión forestal.

Palabras clave: LiDAR, QGIS, GIS, Python 3, Sentinel 2, Segmentación de imágenes, programación.

\begin{abstract}
Sand typing has traditionally been based on cartography and expert criteria of professional foresters. Remote sensing appearance allows obtaining information suitable for automatizing delineation processes. In light of what is previously exposed, this study aims to develop a tool to automatize stand delineation process required in the management of the woods. To achieve that goal, the tool will be developed in Python 3 using as data sources Airborne LiDAR information and Sentinel 2 spatial images. The methodology used, first, processes the LiDAR information, using the FUSION software algorithm, to obtain rasterized information of the vegetation structure variables. Next, a Sentinel-2 derived NDVI raster file is combined with the LiDAR rasters. As a result, a normalized multiband raster (NMR) is generated. Finally, the Orfeo ToolBox Meanshift algorithm is given the NMR as input along with a set of parameters. The software output, a map of the homogeneous forest regions, should not be considered as an ultimate solution, but as a guideline that the forester could use to finally determine forest stands. In order to get better results, for each project and forest type, a different parameter combination has to be trained for every forest type. In addition to the segmentation shapefile, the raster variable files and other byproducts are presented as results. To conclude, the tool presented consists in a modern, free, open data, reliable and fast solution, that combines, algorithms to automatize the first stage of a forest management plan.
\end{abstract}

Keywords: LiDAR, QGIS, GIS, Python 3, Sentinel 2, image segmentation, coding. 


\section{Introducción}

En la actualidad el uso de herramientas informáticas y la automatización de procesos manuales o mecánicos, tanto en la academia como en empresas es una realidad (World Economic Forum, 2018). La creciente preocupación medioambiental y el desarrollo de políticas de gestión sostenible, ha aumentado la demanda de tecnologías de teledetección que sean capaces de proporcionar información y datos para poder cumplir dichas políticas y afrontar los retos medioambientales. Un ejemplo de este uso, es el empleo de Sistemas de Información Geográfica (SIG) y de sensores remotos para ayudar la gestión forestal (White et al., 2016). En el ámbito forestal la incorporación del LiDAR (Light Detection And Ranging) como fuente de datos, ha supuesto una gran revolución para la monitorización de las masas forestales. El LiDAR aéreo (o Aereal Laser Scanner, ALS) ha probado ser efectivo para la adquisición de variables necesarias para el inventario de existencias forestales, principalmente a partir de la altura con errores entre 0.5 a $1 \mathrm{~m}$ (Næsset and Økland, 2002, Wulder et al., 2008). El ALS también puede resultar de interés para obtener otras variables como: volumen, fracción de cabida cubierta o biomasa (Lim et al., 2003). La aparición del LiDAR no es la única tecnología que ha contribuido a facilitar la gestión de las masas forestales, ya que el desarrollo en la carrera espacial y en la tecnología satelital ha permitido, tanto mejorar las telecomunicaciones, como obtener una mayor información sobre nuestros bosques. Esta información es principalmente proporcionada a través de imágenes satelitales obtenidas en las misiones y programas espaciales desarrollados por las agencias espaciales. El programa espacial Copernicus de la Comisión Europea, en la cual la Agencia Europea del Espacio (habitualmente conocida por su acrónimo en inglés: ESA de European Spatial Agency) forma parte, tiene entre sus objetivos desarrollar el programa de satélites Sentinel para monitorizar la tierra y vigilar diferentes aspectos ambientales: calidad de las aguas, limpieza atmosférica, deforestación, erosión, tasa de deshielo, variaciones térmicas (European Spatial Agency, ESA, 2015). Las aplicaciones de esta información proporcionada por la constelación de satélites Sentinel 2 son diversas, una de las más frecuentes es el cálculo de índices de vegetación. Entre ellos, el más utilizado es el índice de vegetación normalizado (NDVI) que ofrece información acerca de la actividad fotosintética de una región (Gandhi et al., 2015). Estas dos tecnologías aunadas con el desarrollo de los sistemas de información geográfica han facilitado el análisis de diferentes tipos de información espacial, permitiendo el desarrollo de gran cantidad de estudios. Hoy en día el uso de la información procesada por los Sistemas de Información Georáfica es prácticamente indispensable para el desarrollo de proyectos de ingeniería. Además, el creciente desarrollo de los lenguajes de programación y los proyectos open source han fomentado la creación y libre distribución, por parte de la comunidad de usuarios y expertos, de una extensa variedad de librerías y softwares para el análisis y estudio de la información geográfica. Gracias a la enorme cantidad de información y de recursos open source disponibles es posible, mediante la combinación de las tecnologías anteriormente mencionadas, 
crear flujos de trabajos automatizados en función de las necesidades analíticas y de trabajo de los usuarios. De esta forma se han reducido el tiempo de procesado de información y aumentado la productividad en los proyectos vinculados a la información geográfica.

Por todo lo anterior, este trabajo de final de máster nace como una propuesta de Agresta S.Coop y la Universidad de Valladolid para contribuir al estudio de las masas forestales creando una herramienta de segmentación automática de "masas homogéneas" a partir de información en abierto proporcionada por sensores remotos. Esta herramienta buscará las zonas homogéneas en masas forestales, contribuyendo a hacer una clasificación previa de tipos de masa, que facilite la preparación del trabajo de campo para una delineación final de tipos de masa.

\section{Material y Métodos}

Esta herramienta emplea las tecnologías anteriormente citadas, y a continuación descritas:

\section{1. $L i D A R$}

El LiDAR (Light Detection And Ranging) es una tecnología que utiliza haces de radiación electromagnética, emitida en pulsos, para obtener la información de posición de un objeto o superficie. La tecnología LiDAR funciona con un sensor activo, es decir, emite su propia radiación electromagnética y no hace uso de fuentes de radiación externa como puede ser un sensor óptico (pasivo). Para obtener la posición de un punto, esta herramienta combina el conocimiento de la posición en cada momento del sensor LiDAR a través de un GPS de alta precisión con el cálculo del tiempo que tarda en volver el haz de luz pulsada, desde que sale del emisor hasta que se refleja en el objeto de interés y vuelve hacia el emisor que lo recibe.

Otra de las características a destacar del LiDAR es que la radiación electromagnética emitida no tiene el mismo comportamiento con todas las superficies. Caben destacar tres casos que en el ámbito forestal nos son de gran ayuda:

- Las superficies solidas generan un único retorno. Ejemplo zonas desarboladas o suelo desnudo.

- El agua no genera un retorno.

- En superficies permeables como cubiertas forestales podemos obtener más de un retorno.

El ALS es masivo y continuo, permitiéndonos conocer la estructura 3D de la vegetación, esta es la principal ventaja que presenta el LiDAR como tecnología para las ciencias forestales. 
En España, la información LiDAR es proporcionada de forma gratuita por el Plan Nacional de Ortofotografia Area - LiDAR (PNOA LiDAR) a través del Centro Nacional de Descargas del CNIG. Actualmente ya está disponible, una primera cartografía competa de todo el territorio nacional con una densidad de puntos de 0.5 puntos por metro cuadrado. La segunda cobertura en desarrollo actualmente proporciona información con densidades que varían desde 0,5 hasta 14 pulsos por metro cuadrado, siendo 1 pulso por metro cuadrado la resolución más generalizada en esta segunda cobertura. Los archivos se distribuyen clasificados, coloreados con las propias fotos del PNOA en formato. laz.

\subsection{Sentinel 2}

El Sentinel 2 es un satélite perteneciente a la familia Sentinel, desarrollada para cumplir exclusivamente las necesidades técnicas y objetivos requeridos por el programa espacial Copernicus de Comisión Europea. En este programa es una actualización del antiguo programa GMES (Global Monitoring for Evironment and Security). El proyecto Copernicus busca, al igual que hacía el GMES, la monitorización de la tierra y servir ante situaciones de emergencia y seguridad ciudadana (European Spatial Agency, ESA, 2015). Respecto a la monitorización del planeta, el proyecto se centra específicamente en: gestión del territorio, medio marino, dinámica atmosférica y calidad y cambio climático. Además, cumple otras misiones como por ejemplo proporcionar información para prácticas agrícolas, forestales, gestión de la seguridad alimentaria. Esta información es esencial para la observación de la evolución de la vegetación en el planeta y todas aquellas aplicaciones relativas al estudio de la vegetación, ya sea a nivel de producción, sanidad, paisaje etc.

Los datos proporcionados por los servicios de Sentinel son ortofotografías aéreas que están distribuidas en el sistema geodésico de referencia WGS84 de

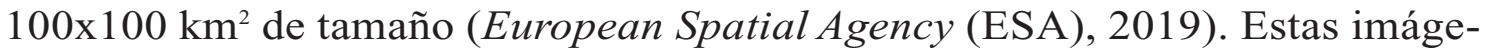
nes están proyectadas en UTM, quiere decir que la tierra está dividida en 60 zonas. Cada zona posee $6^{\circ}$ de ancho longitudinal y $8^{\circ}$ de ancho latitudinal. Las imágenes contienen información referente a determinadas franjas del espectro electromagnético denominadas bandas. Dependiendo de la banda seleccionada poseeremos una resolución espacial (tamaño de pixel diferente), en nuestro estudio, al emplear la B8 y B4 con resolución espacial de $10 \mathrm{~m}$.

\subsection{Sistemas de Información Geográfica: QGIS}

Se seleccionó Quantum Gis para desarrollar el proyecto por su carácter abierto y gratuito. Es un proyecto oficial de la Open Source Geospatial Foundation (OSGeo). Actualmente se encuentra en la versión 3.6 (estable). QGIS ofrece la posibilidad de hacer tus propios desarrollos apoyándose en el lenguaje de programación Python, a través de lo que ellos han denominado PYQGIS, que es la API (Application Programing Interface) de QGIS para ejecutar código Python. (QGIS Development Team, 2018). 
Dentro de OSGeo, también encontramos librerías de manejo de datos espaciales como son SAGA (Conrad et al., 2015), GRASS (GRASS Development Team, 2017) y GDAL (GDAL Developtment Team, 2017) que emplearemos para el procesado de datos, debido a su carácter abierto y su conexión con la interfaz y estructura de QGIS. Adicionalmente, emplearemos Orfeo Tool box para los algoritmos de segmentación de imágenes y FUSION para el procesado de datos LiDAR.

\subsection{Python}

Python es un lenguaje de programación orientada a objetos, interactivo e interpretado. Una de las principales características de Python es su sintaxis clara y su potencia, que lo convierten en un sistema amigable y de fácil uso para el usuario novel en la programación.

Una vez introducidos los materiales, se presenta la metodología que se resume en el siguiente flujo de trabajo que la herramienta debe automatizar. Podemos resumir la metodología en 4 partes: Procesado LiDAR, Procesado Sentinel, Procesado Raster y Segmentación y suavizado.

\subsection{Procesado LiDAR}

La rama derecha del diagrama de flujo (Figura 1) aborda el procesado de los datos LiDAR a través de la herramienta FUSION (McGaughey, 2018). La herramienta desarrollada en una primera fase, comienza realizando el procesado de los datos llamando a FUSION. Primero, transforma los archivos LiDAR en formato ".laz" en un modelo digital de elevaciones (MDE) en formato ".dtm" propio de Fusion. Para ello el usuario, especificará las clases que querrá procesar (por defecto opera con la clase 2 para el suelo) y el tamaño de pixel (recomendable como mínimo $2 \mathrm{~m}$ para PNOA, $0,5 \mathrm{pulsos} / \mathrm{m}^{2}$ ). Una vez generado el MDE hay que convertirlo a un formato raster de intercambio de archivos ".asc" para poder cargarlo en cualquier SIG convencional. Los MDE resultantes se obtienen en formato ".asc" y con una resolución de $2 \times 2 \mathrm{~m}$.

Una vez obtenidos los MDE, la herramienta calcula las métricas en continuo de la nube de puntos LiDAR en celdas de un tamaño dado para cada bloque LAZ (GridMetrics). Para ello se le deberán indicar a la herramienta las clases a procesar, que en nuestro casi serán las correspondientes a vegetación y suelo $(2,3,4,5)$, dejando al margen aquellos puntos que no pertenezcan a dichas clases. Además, se podrán definir el tamaño de pixel, la altura mínima de procesado de alturas y coberturas. El resultado de esta operación son dos archivos de texto ".csv", uno que recoge las métricas calculadas a los retornos en función de su altura y otro en función a su intensidad. Dentro de las métricas por elevación encontramos una serie de variables, que tienen correlación con estructura de la vegetación, en concreto para este desarrollo hemos elegido las siguientes métricas:

1. Elevación P90: elevación a la que se recoge el 90 por ciento de los retor- 


\section{Flujo de Trabajo de la Herramienta de Segmentación}

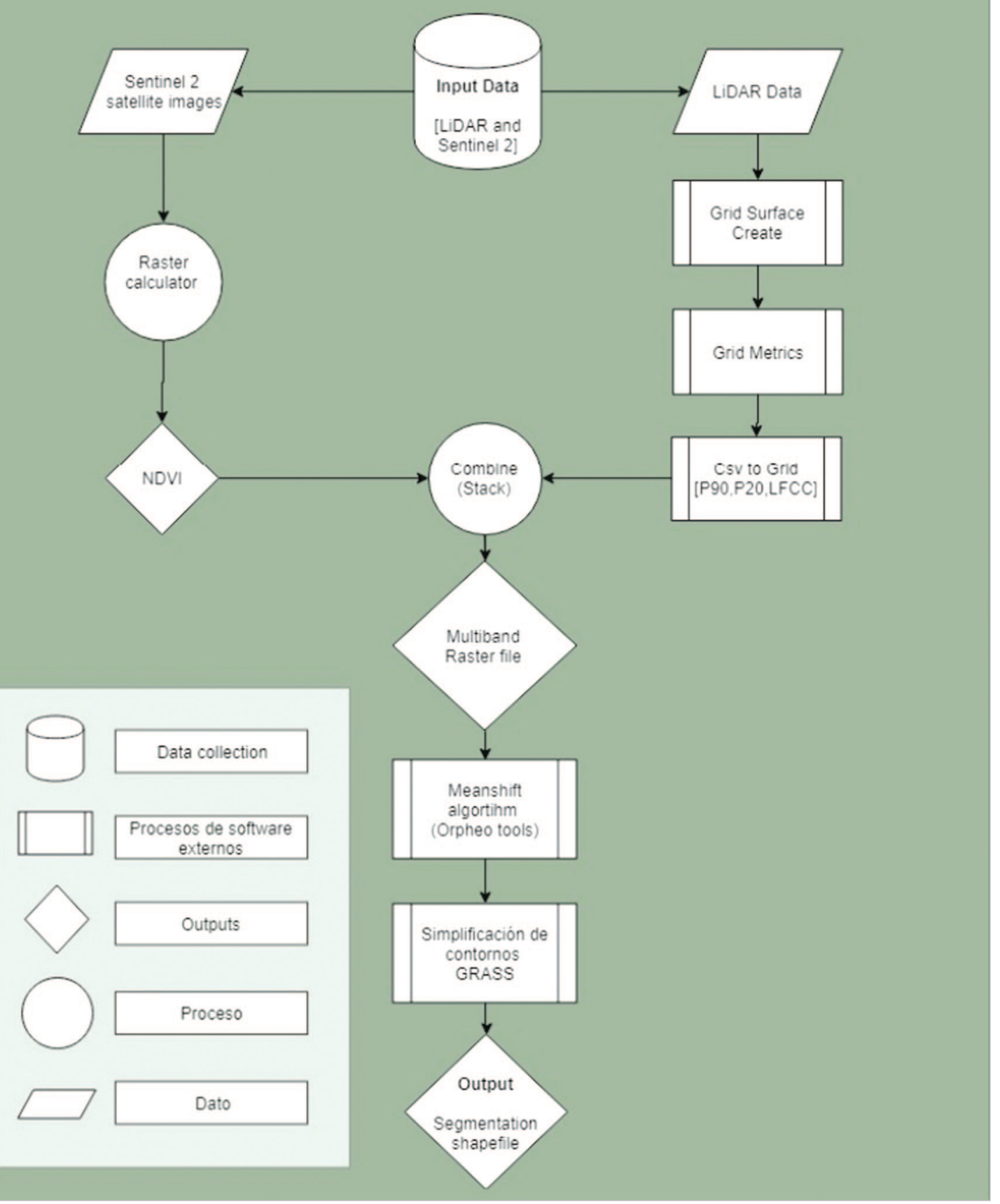

Figura 1. Flujo de trabajo,

nos de un pulso. Esta variable está muy correlacionada con la altura dominante de la vegetación.

2. Elevación P20: elevación a la que se recoge el 20 por ciento de los retor- 
nos de un pulso. Aunque de un modo simplista esta variable tiene una cierta relación con la altura base de copa.

3. Porcentaje de primeros retornos por encima de la altura de corte: Se relaciona con la fracción de cabida cubierta.

Mediante algoritmos de Fusion (CSV2Grid) extraemos estas variables de formato de texto a formato ráster ".asc" y posteriormente el desarrollo une los raster generados por bloques laz individuales en un único ráster .tif (algoritmo GDAL:merge (Warmerdam et al., 2019)) para cada variable que represente la zona de estudio.

\subsection{Procesado de Sentinel}

En la rama de izquierda del diagrama (Figura 1) se muestra el procesado de las escenas obtenidas con Sentinel 2. Empezaremos calculando el índice de vegetación normalizado (NDVI) que se define con la siguiente expresión (a la derecha bandas empleadas en Sentinel 2):

$$
\mathrm{NDVI}=\frac{(N I R-R O J O)}{(N I R+R O J O)}=\frac{(B 8 a-B 4)}{(B 8 a+B 4)}
$$

Este índice muestra la actividad fotosintética de la vegetación y se mueve en un rango de $[-1,1]$ siendo 1 la mayor actividad fotosintética. Este índice aprovecha la alta diferencia de reflectancia de la vegetación fotosintéticamente activa, para las longitudes de onda comprendidas en el rango del infrarrojo cercano y del rojo. Es decir, la vegetación no fotosintéticamente activa (carente de clorofila) quedará reflejada en este índice con valores bajos.

Para obtener el índice de vegetación normalizado, recurrimos al algoritmo de GRASS vegetation index (Kamble \& Chemin, 2018). Este algoritmo está integrado en QGIS y por lo tanto no ofrece problemas de compatibilidad. Al igual que el que desarrollamos, este algoritmo emplea la misma fórmula anteriormente expuesta.

El resultado de este procesado es un ráster “.tif” de gran tamaño (100x100 $\mathrm{km}^{2}$ ), que excede el tamaño del área de estudio. Para ajustarla al área de estudio, el ráster de NDVI generado se recorta usando como límite la extensión de la capa ráster generada a través del procesado LiDAR empleando GDAL:cliprasterbyextent (QGIS Development Team, 2019). Se ha seleccionado GDAL por tratarse de una librería abierta y gratuita instalada por defecto dentro de QGIS, de esta forma evitamos errores e incompatibilidades.

\subsection{Procesado Ráster}

Previamente a la creación del ráster multibanda se deberán normalizar las variables rasterizadas. Esto quiere decir que, para poder ser comparables dichas va- 
riables, éstas deben estar dentro del mismo rango de valores. El rango de valores elegido, es de 0 a 100 . Los valores nulos o los no data serán reclasificados con el valor -9999 .

Dicha reclasificación se ha programado específicamente para cada variable:

1. Para la variable, fracción de cabida cubierta, no se han realizado modificaciones, puesto que, por defecto, esta capa ya esta escalada de 0 a 100 .

2. Las capas relacionadas con las variables de alturas de la vegetación (P90 y P20), se calculó:

O Para cada pixel de la capa < percentil 90 la siguiente expresión:

$$
\text { valor normalizado }=\frac{\text { valor del pixel }-2}{\text { percentil } 90 \text { de la capa }-2} * 100
$$

En lugar de emplear el valor máximo de la capa para normalizar se emplea el percentil 90. Esto se justifica porque se han detectado errores de clasificación en los datos LiDAR del PNOA en las zonas piloto. Estos errores, suponen que sea posible encontrar, puntos de alturas máximos que no se corresponden con la vegetación, como por ejemplo una catenaria o un edificio. Se resta dos porque es la mínima altura de procesado del LiDAR. Así hacemos que las curvas tengan de origen 0 .

$$
\begin{aligned}
& \text { Para pixeles cuyo valor }>\text { P90: } \\
& \text { valor normalizado }=100
\end{aligned}
$$

Se considera que aquellos valores por encima del percentil indicado corresponden con elemento que está por encima de la altura de la vegetación y se reclasifica como 100, con el máximo valor de altura.

3. El índice de vegetación ha sido normalizado teniendo en cuenta únicamente los valores entre 0 y 1 , los cuales representan a la vegetación fotosintéticamente activa (todo aquel valor menor que cero se considera 0 ). A estos valores se les ha aplicado la siguiente reclasificación:

$$
\text { valor normalizado }=\frac{\text { valor del pixel }}{\text { valor máximo de la capa }} * 100
$$

Una vez obtenidos los resultados del procesado LiDAR y de Sentinel 2 y normalizadas las variables, procedemos a crear un ráster multibanda que recopile la información de todas las capas haciendo un stack (o pila) de capas. Como resultado final, obtenemos un ráster multibanda en formato tif, que recoge la información, tanto del LiDAR como del Sentinel en una sola imagen. En la figura 2 se puede muestra la distribución de las bandas agrupadas en el raster final: 


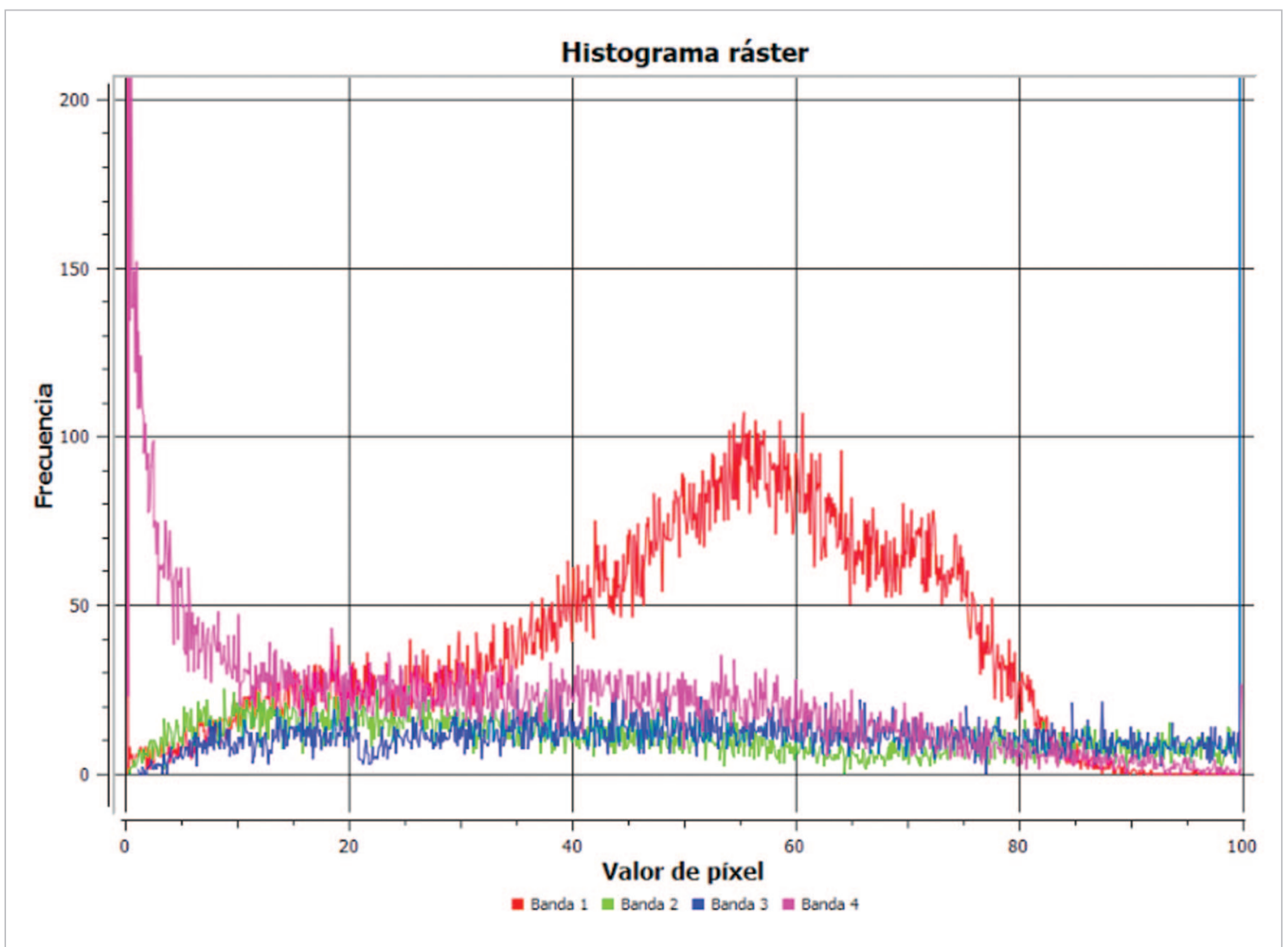

Figura 2. Histograma de frecuencias para las bandas del ráster multibanda. Banda 1: NDVI, banda 2: P20, banda 3: P90, banda 4: LFCC.

\subsection{Segmentación con Segmentation (Meanshift) OTB}

Empleamos el ráster multibanda como input para el algoritmo de segmentación meanshift, disponible en la caja de herramientas Orpheo Tool Box, OTB (OTB-Team, 2018). Este algoritmo funciona uniendo aquellos pixeles de una imagen multiespectral que se encuentren a un rango de distancia inferior a un rango de radio (y opcionalmente a la distancia espacial por debajo del rango espacial) formando así un clúster (Youssefi, 2018).

Hay dos algoritmos de segmentación disponibles, versiones diferentes del algoritmo de segmentación meanshift, el primero se fundamenta en componentes conectados basados en píxeles simples según un criterio definido por el usuario (generalmente la distancia), mientras que el segundo, watershed se basa en el gradiente de intensidad. Dado el propósito de selección de áreas homogéneas, en nuestro caso emplearemos el primero.

El algoritmo de segmentación de OTB ofrece con dos vías principales de operar que condicionan el archivo de salida:

- Modo ráster: devolverá un ráster en el que los pixeles vecinos son clasificados con la misma etiqueta, es decir devuelve una segmentación de eti- 
quetas y colores. Este modo carga toda la imagen a la memoria, por lo tanto, no puede procesar grandes escenas.

- Modo vectorial: En este modo se devolverá una capa shapefile. En esta capa se crearán teselas segmentadas de acuerdo a los parámetros y algoritmos establecidos por el usuario. En este modo se hace un uso más eficiente de la memoria, de tal forma que se pueden procesar imágenes de mayor tamaño. Finalmente, al estar activada la opción stitch se unirán los segmentos separados accidentalmente por el esquema de teselado. Esta segunda vía se ha considerado más eficiente para el objetivo final del proyecto, siendo la seleccionada para herramienta programada.

El algoritmo se configura a través de la selección de los siguientes parámetros por parte del usuario:

- Spatial radius: radio espacial de los vecinos o el umbral en distancia espacial para considerar píxeles en el mismo segmento (Youssefi, 2018). Valor por defecto del algoritmo: 5.

- Range radius: Rango de radios que definen el radio en el espacio multiespectral expresado en unidades radiométricas. También puede definirse como el umbral en la distancia euclidiana de la firma espectral (expresado en unidad de radiometría) para considerar los píxeles en el mismo segmento (Youssefi, 2018). Valor por defecto del algoritmo: 5.

- Maximum number of iterations: El algoritmo iterativo terminará las convergencias una vez se haya alcanzado el número máximo de iteraciones. Valor por defecto del algoritmo: 100.

- Minimum region size: Tamaño mínimo de una región en la segmentación (en pixeles). Los clústeres más pequeños se fusionarán con los clústeres vecinos adyacentes con la radiometría más cercana. Tamaño mínimo de región es el tamaño del pixel. Valor por defecto del algoritmo: 100.

El resultado será un archivo vectorial en formato shapefile que, cumpliendo con las condiciones anteriores, haya realizado una segmentación del monte. Cabe destacar que para optimizar la segmentación en cada escena o cada área de estudio los valores por defecto de la herramienta serán diferentes (en cuanto a tamaño de los segmentos, por valores de las bandas de la imagen multiespectral, texturas, tamaño total del área de estudio...), por lo que conseguir los mejores resultados es aconsejable que el usuario se identifique tanto con la herramienta como con los parámetros utilizados. Normalmente, la segmentación por defecto es útil como referencia a un segundo intento de segmentación, si el resultado con los valores por defecto no ha sido satisfactorio. Con un poco de práctica, el usuario podrá llegar a realizar segmentaciones satisfactorias al primer intento. 


\subsection{Suavizado de contornos Chaiken algorithm (GRASS)}

La segmentación realizada por meanshift nos devolverá un archivo con esquinas muy angulosas y de un aspecto artificial, por eso aplicaremos el último paso antes de obtener el producto final. Este paso consiste en un suavizado de vértices, que funciona de forma inversa a la generalización de contornos (reducción del número de vértices).

El algoritmo de Chaiken suaviza una curva mediante la sustitución iterativa de cada punto por dos nuevos puntos: uno a $1 / 4$ del camino al siguiente punto $\mathrm{y}$ otro a 1/4 del camino hasta el punto anterior, así "complica" la geometría aumentando sus elementos y por lo tanto su complejidad. Este algoritmo se caracteriza por generar una nueva línea que inscriba la original, de tal forma que los puntos de esta nueva están a una distancia umbral de la original (Bundala et al., 2007). Empleamos el algoritmo de Chaiken implementado dentro de GRASS (y por lo tanto de QGIS) bajo el nombre de grass7:v.generalize (identificador del algoritmo en QGIS 3).

\subsection{Entregables de la herramienta}

Al finalizar el flujo de trabajo la herramienta devolverá automáticamente los siguientes resultados:

a. Un modelo digital de elevaciones del terreno LiDAR en formato ASCII.

b. Tres rastres (ASCII) que representan diferentes variables forestales, por defecto la herramienta ofrece:

a. Una aproximación a la altura dominante.

b. Una aproximación a la altura base de copa.

c. Fracción de cabida cubierta.

c. Un ráster con el índice de vegetación normalizado para la zona de estudio.

d. Una segmentación preliminar sin un suavizado de bordes.

e. Por último, a propuesta de los rodales homogéneos con un suavizado de bordes (Chaiken y Snakes).

\subsection{Test de la herramienta}

Con objeto de poner a punto la herramienta se han seleccionado dos zonas piloto con distintas características: una zona eminentemente forestal de la Sierra de Guadarrama (Madrid) y un monte periurbano en el entorno de la ciudad de Madrid. 
La primera zona piloto se encuentra enmarcada dentro de la Comunidad de Madrid, en concreto en la zona norte de la Sierra de Guadarrama: Cabecera del valle de Lozoya, montes de Cabeza de Hierro y Peñalara (Figura 3). Se trata de un bosque de coníferas de alta montaña dominada principalmente por Pinus sylvestris; L. Las formaciones vegetales de esta zona piloto se caracterizan por ser bosques monoespecíficos cerrados (fracciones de cabida cubierta elevada) en los cuales sus pies alcanzan alturas alrededor de los 20-25 metros. Pudiendo darse un subpiso de Pteridium aquilinum con pies aislados de alguna especie acompañante como Taxus baccata, Ilex aquifolium.

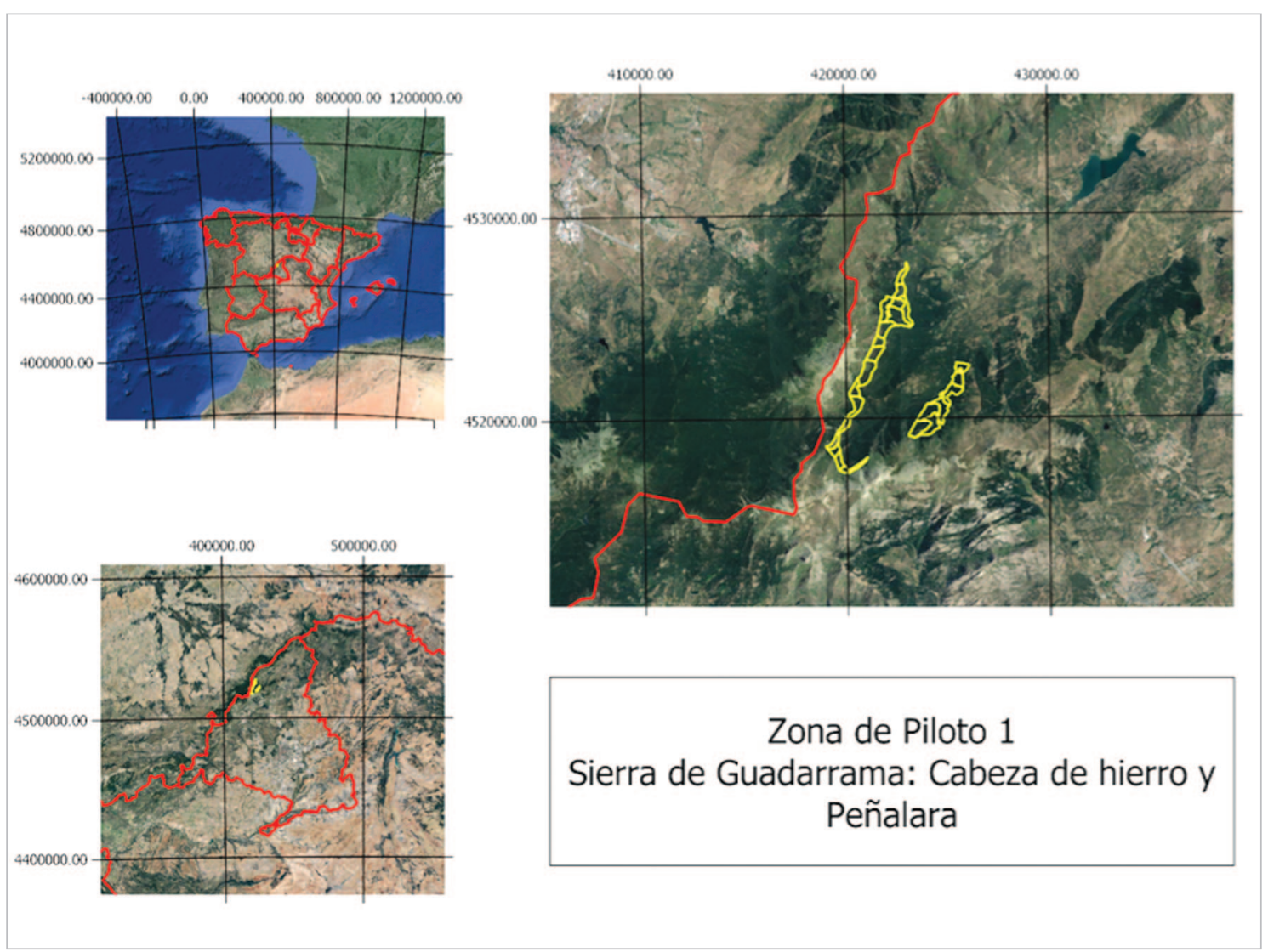

Figura 3. Zona piloto 1. Comunidad de Madrid. Sierra de Guadarrama: Peñalara y Cabeza de Hierro.

La zona piloto 2 es un monte mediterráneo mixto de Pinus halepensis. L. y Quercus ilex ballota emplazado al norte de la ciudad de Madrid (Valdelatas). En esta zona piloto a diferencia de la primera, encontramos un bosque de menores cobertura, densidad y alturas, con espacios abiertos y dos estratos claramente diferenciados entre el pino (dominante) y la encina.

Realizar el estudio sobre estas dos zonas nos ha permitido observar cómo se comporta la herramienta frente a dos tipologías de masas completamente diferentes. En las figuras 5 y 6 se pueden apreciar tanto los resultados de segmentación obtenidos como los parámetros seleccionados para cada una de las zonas piloto. 


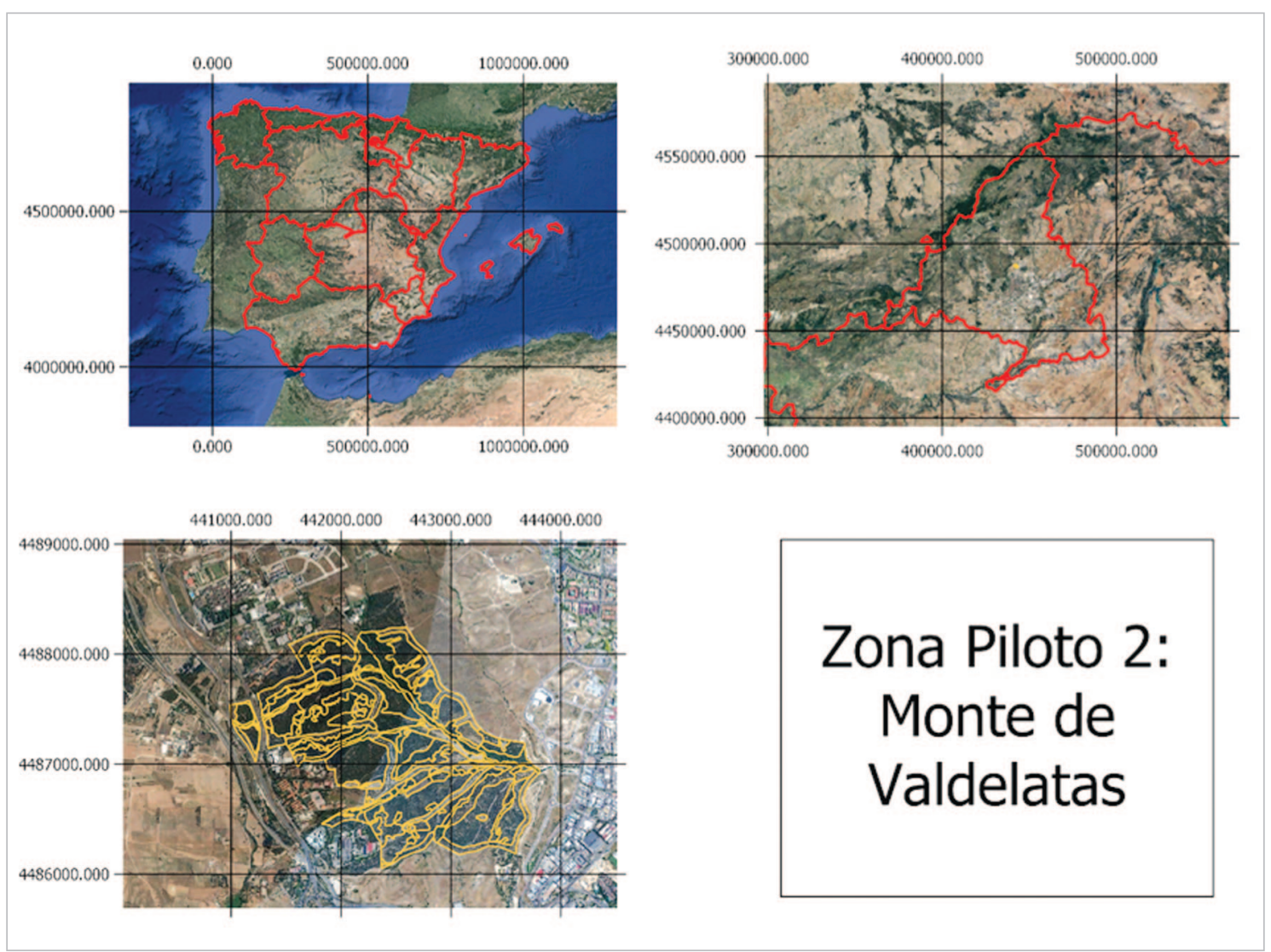

Figura 4. Zona piloto 2. Bosque periurbano: Monte de Valdelatas.

\section{Resultados}

Los resultados obtenidos tanto en zonas heterogéneas como en zonas forestales con densidades medias se consideran satisfactorios. En las áreas donde, la diversidad de masas forestales sea mayor (y preferiblemente el tamaño sea menor), como en Valdelatas, la herramienta podrá dar un resultado más aproximado a la segmentación final que desea el usuario. Sin embargo, en aquellas zonas homogéneas con coberturas altas, será necesario prestar una mayor atención a la configuración del algoritmo meanshift y emplear zonas de estudio de menor tamaño, o fragmentar la zona en unidades a procesar individualmente. En áreas homogéneas es aconsejable el empleo de la herramienta con valores de los parámetros de entrada por defecto, de esta forma, el resultado de la segmentación será más grosero en cuanto a número de divisiones, pero con un mayor sentido biológico. Esto posibilita que la herramienta se pueda usar como alternativa para generar unidades del paisaje en este tipo de estructuras. Posteriormente el usuario podría detallar manualmente, aquellas regiones de interés que no obtuvieron el resultado esperado. 


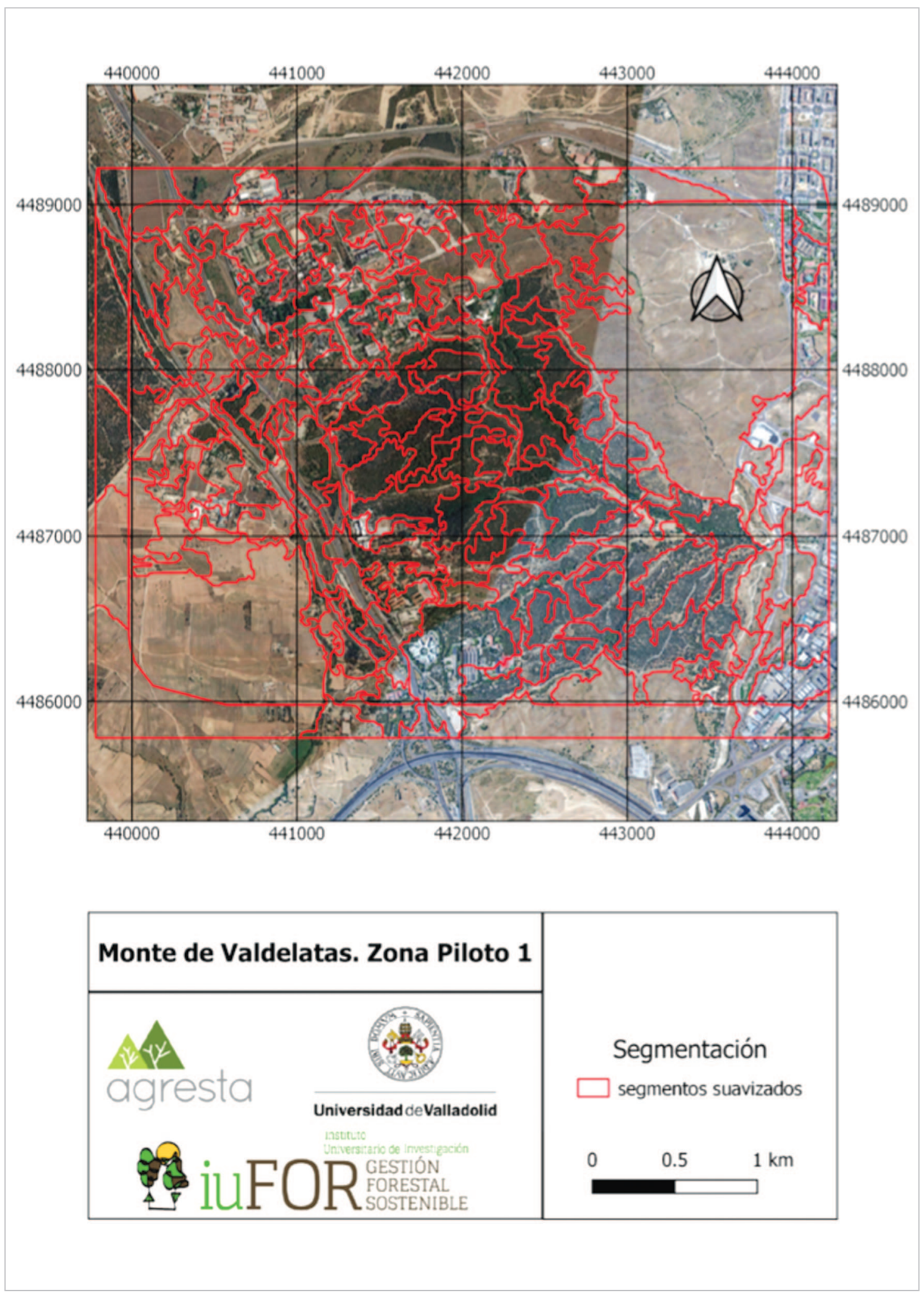

Figura 5. Segmentación con algoritmo meanshift. Variables Spatial radius: 4 Range Radius: 12, Maximun iteration number: 150 , Minimun region size: $80 \mathrm{~m}$. 


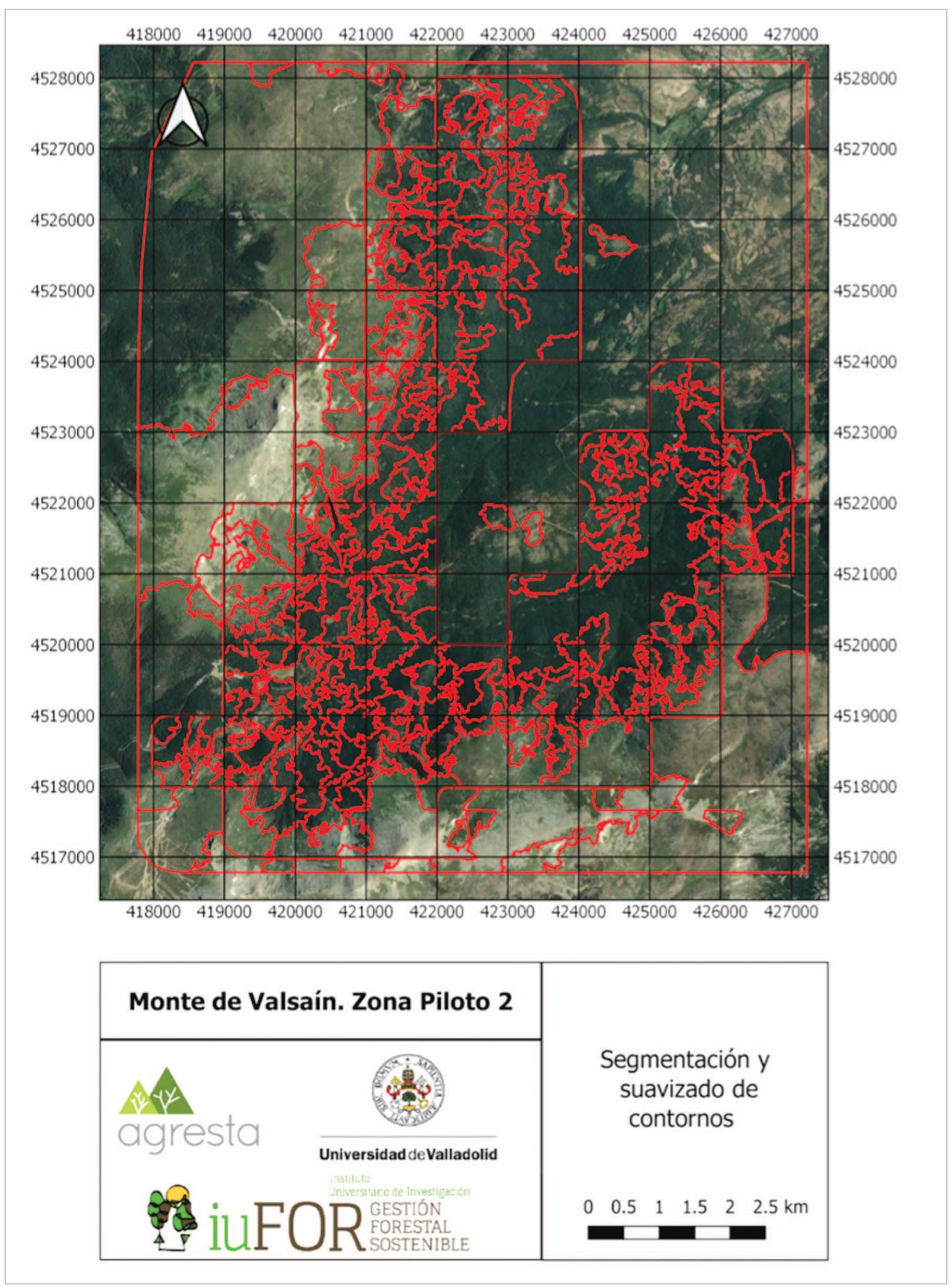

Figura 6. Propuesta de rodalización con simplificación de contornos sobre Sierra de Guadarrama: Peñalara y Cabeza de Hierro. La foto está superpuesta a la imagen satelital ofrecida por Bing Sat para una mejor interpretación. Variables Spatial radius: 5 Range Radius: 10, Maximum iteration number: 150, Minimum region size: $100 \mathrm{~m}$. 


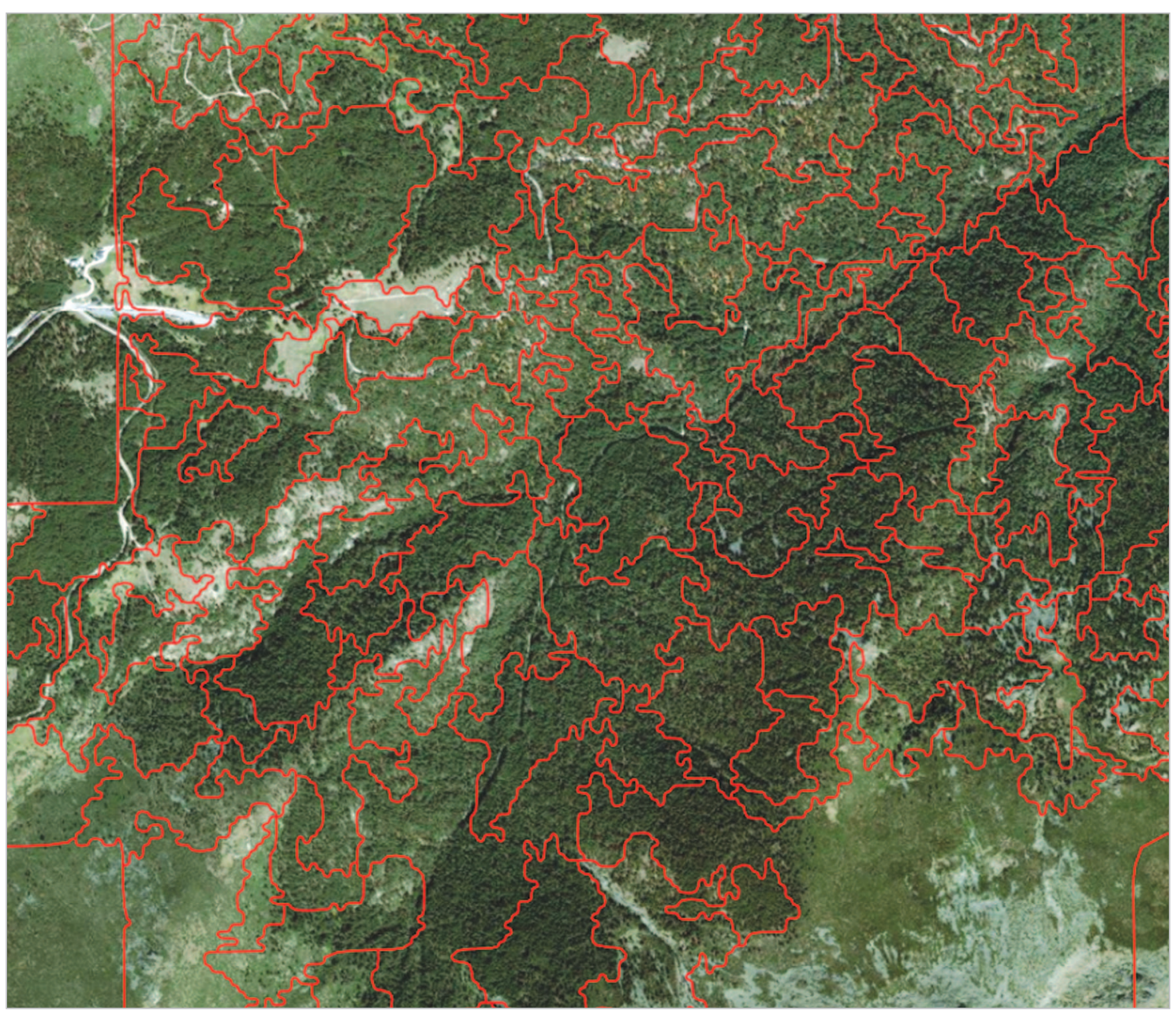

Figura 7. Detalle de la propuesta de rodalización para la zona piloto de la Cabecera del valle de Lozoya.

\section{Discusión y Conclusión}

La metodología desarrollada se inspira en proyectos parecidos de automatización de datos LiDAR, en los cuales se han desarrollado metodologías relativamente similares que han obtenido reconocimiento entre la comunidad forestal, como en la herramienta SilviLiDAR (Crespo \& Díez Rabanos, 2018). Este proyecto, además del procesado utiliza la información generada para proponer medidas de gestión orientadas a las distintas situaciones selvícolas identificadas para cada zona. En la herramienta propuesta en este estudio, se trabaja con segmentación orientada a objetos en lugar de pixel a pixel, incluyéndose además el uso de las imágenes Sentinel como dato de entrada en la segmentación, lo que supone complementar a la información de estructura proporcionada por el LiDAR con información espectral del satélite a la hora de alimentar el algoritmo de segmentación. A diferencia de SilviLiDAR la presente herramienta no propone medidas de actuación silvícola asociadas a los distintos segmentos detectados. 
En otros trabajos (Cantero Fauquier, 2016), se plantean metodologías enfocadas a modelos de árbol individual, donde se procesan los datos LiDAR para la delineación de copas a partir de la generación de Modelos de altura de vegetación con y sin el aporte de ortofoto. Sin embargo, se trata de un flujo de trabajo de carácter semiautomático que carece del componente de automatización, que ofrecen tanto SilviLiDAR o en esta herramienta. Por otro lado, su utilización no genera los subproductos (por ejemplo, ráster con variables LiDAR) orientados a la planificación y gestión forestal que complementan el producto desarrollado. Este tipo de trabajos se centran más en observar el resultado de la segmentación sobre determinado tipo de cubiertas forestales, mientras que este proyecto se presenta como una herramienta de gestión.

Una de las ventajas de la herramienta es su accesibilidad al usuario, ya que está desarrollada a partir de datos y software gratuitos y en su mayoría abiertos (excepto FUSION, pero que también es gratuito). Además, el script está implementado sobre el API (Application Programming Interface) de QGIS y utiliza de sus librerías. Esta característica permite, que la herramienta sea fácilmente distribuible y accesible por otros usuarios, ya que la gran mayoría de funciones requeridas por el programa está incluida en QGIS y no requieren de gran cantidad de instalaciones paralelas de programas o librerías.

El objetivo de este estudio es el crear una herramienta informática que genere unidades homogéneas del monte, a partir de datos Satelitales y LiDAR que, faciliten el trabajo de teselado que requiere cualquier planificación forestal. La herramienta genera de una manera ágil y rápida una rodalización previa como base para el trabajo de campo. De esta forma, el gestor ahorra tiempo de gabinete en el proceso de delimitación de zonas homogéneas en la zona de estudio ganando precisión al mismo tiempo. El resultado generado, está llamado a ser una herramienta muy potente para acabar de afinar los límites reales de los tipos de masa durante el trabajo de campo, un trabajo de campo que puede ser más intenso merced al tiempo que ahorra este script en la preparación del mismo en gabinete.

\section{Bibliografía}

A Wulder, M.; W. Bater, C.; C. Coops, N.; Hilker, T.; \& C White, J.; 2008. The role of LiDAR in sustainable forest management. The Forestry Chronicle, 84(6), 807-826. Retrieved from http://pubs.cif-ifc.org/doi/abs/10.5558/tfc84807-6 https://doi.org/10.5558/tfc84807-6

Bundala, D.; Bergenheim, W.; \& Metz, M.; 2007. GRASS: v.generalize. Retrieved from https: //grass.osgeo.org/grass77/manuals/v.generalize.html

Cantero Fauquier, F.J.; 2016. Generación de herramientas de localización de árboles individuales con el módulo de procesado de QGIS a partir de información LiDAR y ortofotografía aérea.Tesis de Fin de Máster. Universidad Politécnica de Madrid

Conrad, O.; Bechtel, B.; Bock, M.; Dietrich, H.; Fischer, E.; Gerlitz, L.; Böhner, J.; 2015. System for Automated Geoscientific Analyses (SAGA). Geosci. Model Dev., 8, 1991-2007. https:// doi.org/10.5194/gmd-8-1991-2015 
Crespo Rodrigo, A.; Díez Rabanos, F.J.; 2018. SilviLIDAR: Herramienta de manejo de datos LiDAR para la gestión forestal. Montes, 134, 23-26.

European Spatial Agency (ESA), 2015. Overview / Copernicus / Observing the Earth / Our Activities / ESA. Retrieved February 18, 2019, from https://www.esa.int/Our_Activities/Observing_the_Earth/Copernicus/Overview3

European Spatial Agency (ESA), 2019. Sentinel-2 - Data Products - Sentinel Handbook. Retrieved February 19, 2019, from https://sentinel.esa.int/web/sentinel/missions/sentinel-2/dataproducts

GDAL Development Team, 2017. GDAL - Geospatial Data Abstraction Library. Open Source Geospatial Foundation.

GRASS Development Team, 2017. Geographic Resources Analysis Support System (GRASS). Open Source Geospatial Foundation.

McGaughey, R.J.; 2018. FUSION/LDV: Software for LIDAR Data Analysis and Visualization. 214 p Seattle: U.S. Department of Agriculture, Forest Service, Pacific Northwest Research Station, University of Washington.

Næsset, E.; \& Økland, T.; 2002. Estimating tree height and tree crown properties using airborne scanning laser in a boreal nature reserve. Remote Sensing of Environment, 79(1), 105 -115. https://doi.org/10.1016/S0034-4257(01)00243-7

OTB-Team, 2018. Segmentation - Segmentation. Retrieved from https://www.orfeo-toolbox.org/ CookBook/Applications/app_Segmentation.html

QGIS Development Team, 2018. QGIS Geographic Information System. Open Source Geospatial Foundation Project. Retrieved from http://qgis.osgeo.org"

QGIS Development Team, 2019. Clip raster by extent. Retrieved from https://docs.qgis.org/2.8/ en/docs/user_manual/processing_algs/gdalogr/gdal_extraction/cliprasterbyextent.html

Warmerdam, F.; Rouault, E.; \& Others, A.; 2019. GDAL: gdal_merge.py. Retrieved from https: //gdal.org/gdal_merge.html

White, J.C.; Coops, N.C.; Wulder, M.A.; Vastaranta, M.; Hilker, T.; Tompalski, P.; 2016. Remote Sensing Technologies for Enhancing Forest Inventories: A Review. Canadian Journal of Remote Sensing, 42(5), 619-641. https://doi.org/10.1080/07038992.2016.1207484

World Economic Forum, 2018. The Future of Jobs Report. Executive Summary (Vol. 5). https:// doi.org/10.1177/1946756712473437

Youssefi, D.; 2018. LSMSSegmentation - Exact Large-Scale Mean-Shift segmentation, step 2. OTB CookBook. Retrieved from https:/www.orfeo-toolbox.org/CookBook/Applications/ app_LSMSSegmentation.html 
